\title{
Serial PEF measurement is superior to cross-shift change in diagnosing occupational asthma
}

\author{
D. Park*, V.C. Moore*, C.B.S.G. Burge*, M.S. Jaakkola*,\#, \\ A.S. Robertson ${ }^{\star, \#}$ and P.S. Burge ${ }^{\star, \#}$
}

ABSTRACT: Cross-shift measurements of peak expiratory flow (PEF) are commonly employed in the diagnosis of occupational asthma, although evidence for this approach is lacking. The current paper presents an evaluation of the technique.

Mean changes in PEF across morning/day shifts were compared between workers with occupational asthma, confirmed using specific challenge testing, and non-working asthmatics. Individuals were divided into a development set, used to identify the optimum cross-shift change for diagnosing occupational asthma, and an evaluation set, used to test the sensitivity and specificity of this value. Comparative analysis of serial PEF records was performed using the Oasys-2 computerised system.

A cross-shift decrease in PEF of $5 \mathrm{~L} \cdot \mathrm{min}^{-1}$ achieved acceptable specificity in the development set. Applied to the evaluation set, this cut-off had a specificity of $90.9 \%$ and a sensitivity of $50 \%$. Sensitivity could not be improved without unacceptable compromise to specificity. Analysis of serial PEF records using linear discriminant analysis identified occupational asthma with a sensitivity of $\mathbf{8 3 . 3} \%$ and a specificity of $\mathbf{9 0 . 9 \%}$. Serial analysis using mean work/rest day PEF comparison had a sensitivity of $66.7 \%$ and a specificity of $100 \%$.

Cross-shift changes in PEF in morning/day-shift workers have poor sensitivity in diagnosing occupational asthma, and are inferior to serial techniques.

KEYWORDS: Oasys-2, occupational asthma, peak expiratory flow, pulmonary function testing

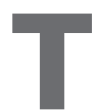
he accurate diagnosis of occupational asthma can prove challenging. A history of symptoms that improve on days away from work has acceptable sensitivity but poor specificity [1, 2]. Objective tests to confirm the diagnosis are therefore required. There is some evidence related to the serial measurement of peak expiratory flow (PEF) (sensitivity 81.8\%, specificity $93.8 \%$ [3]) and changes in nonspecific airway reactivity at and away from exposure (sensitivity $48-67 \%$, and specificity $54-78 \%[4,5]$ ) as acceptable confirmatory tests. Specific inhalation testing remains the gold standard, but suffers from a general lack of availability and more complex exposures may prove difficult to reproduce. Many health practitioners use a simple comparison between pre- and post-shift lung function measurements. Cross-shift changes have also been used widely in epidemiological studies of occupational asthma [6-9]. Individuals with occupational asthma are presumed to have a significant fall between pre- and post-shift measurements. A recent evidence-based review of the management of occupational asthma by the British Occupational Health Research Foundation (BOHRF) has identified a paucity of evidence for simple cross-shift measurements in the diagnosis of this condition [2].

We carried out a study attempting to measure the diagnostic accuracy of cross-shift PEF measurements in the identification of individuals with occupational asthma, using specific inhalational challenge testing as the reference standard. This was compared with two methods of serial analysis from the computer-based Oasys-2 system (available from www.occupationalasthma. com) [10].

\section{METHODS}

Workers were identified retrospectively for the study from a comprehensive computerised database of individuals attending a tertiary centre respiratory clinic in the UK. All had been referred for investigation of possible occupational asthma. Whilst attending the clinic, serial PEF recordings
AFFILIATIONS

*Occupational Lung Disease Unit, Birmingham Heartlands Hospital, and \#Dept of Occupational and Environmental Medicine, University of Birmingham, Edgbaston, Birmingham, UK.

CORRESPONDENCE

P.S. Burge

Occupational Lung Disease Unit Birmingham Heartlands Hospital Bordesley Green East Birmingham B9 5SS

UK

E-mail: sherwood.burge@ heartofengland.nhs.uk

Received:

Oct 232008

Accepted after revision:

March 092009

First published online:

March 262009 
had been performed as part of the patients' diagnostic workup. Individuals were asked to measure their PEF at 2-h intervals over a 3-week period, including both work days and days not at work ("rest days"). An example of a PEF record from an individual, with readings for work and rest days is shown in figure 1.

All individuals with positive results from specific inhalation challenge testing, who had completed a serial PEF record while exposed at work were identified for possible inclusion in the study. In order to allow a sensible comparison to be made between the cross-shift and the serial methods of analysis using Oasys- 2 , records were required to contain: $\geqslant 2$ rest days; $\geqslant 3$ workdays whilst exposed to the relevant agent; $\geqslant 4$ readings per day for $\geqslant 75 \%$ of days in the record; and at least one reading available in the hour before starting work on all workdays. Only records covering morning or day shifts were considered. Start times for these shifts ranged between 04:00 h and 11:00 h. Only one record was used per worker. The computerised database was searched between 1990 and 2005, although no suitable subjects were identified with serial PEF recorded prior to 1993. In total, there were 51 individuals with both a positive specific challenge, and serial PEF data from a period whilst exposed to the appropriate agent. The serial PEF records of 36 of these individuals were sufficiently comprehensive to meet the criteria for inclusion, and formed the occupational asthma group.

A control group was drawn from attendees at the same clinic who had been diagnosed with asthma on clinical grounds, and who had serial PEF recordings available from a period when they were not at work. Records for inclusion in the control group were required to meet the same inclusion criteria as the

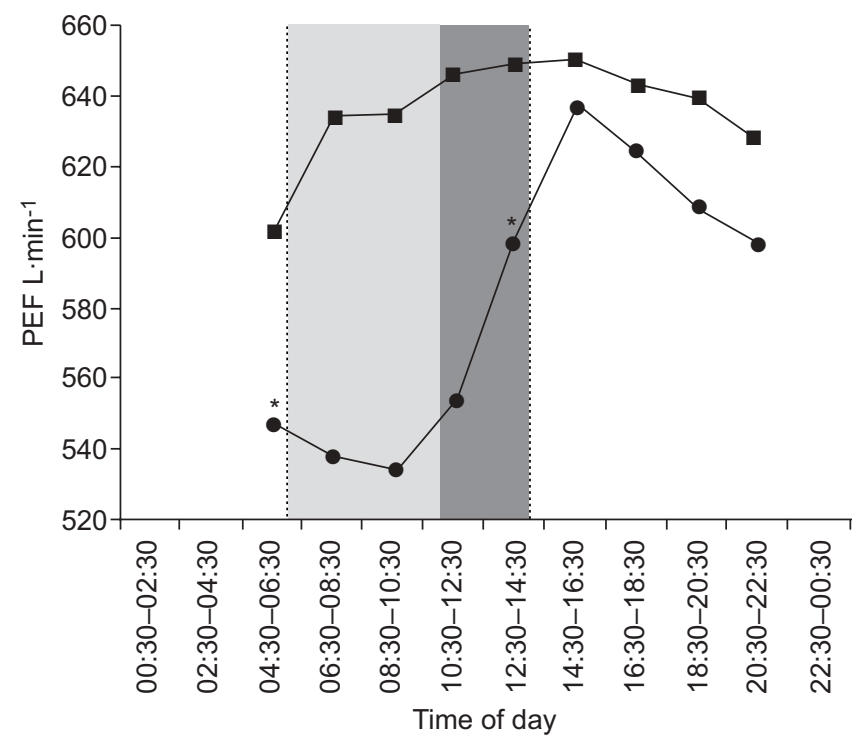

FIGURE 1. A 2-hourly plot of peak expiratory flow (PEF) using the Oasys program (available from www.occupationalasthma.com), with readings for rest ( and work (-) days. A cross-shift calculation of PEF for this individual would be measured between the two asterixed readings, and show an increase of $32 \mathrm{~L} \cdot \mathrm{min}^{-1}$. The shaded areas show the time working. All working days started at 06:00 h; the mode and latest stopping work time was 14:00 h, and the earliest stopping work time was 11:00 $\mathrm{h}$ (the left margin of the darker shaded area). occupational asthma group. To allow cross-shift and serial PEF analysis, an arbitrary "work" period from 09:00-17:00 h Monday to Friday was superimposed over the control group records. Between 1989 and 1999 there were a total of 60 PEF records available from a period when the individuals concerned were not at work. Of these records, 44 were sufficiently comprehensive to make up the control group.

The occupational asthma and control groups were divided equally between a development set and an evaluation set. Allocation was carried out alternately in chronological order of presentation.

Cross-shift comparisons were made by subtracting the daily post-shift reading (taken as the last reading at work after a minimum of $4 \mathrm{~h}$ at work) from the pre-shift value (defined as the last morning recording available in the hour before starting work) and then calculating the mean value.

A cut-off value was derived from data in the development set. In order that this value should allow confident diagnosis of occupational asthma, a decision was made a priori that it should have $\geqslant 95 \%$ specificity. The optimum cut-off to achieve this was identified using a receiver operating characteristic (ROC) curve. The cut-off value was tested against the individuals in the evaluation set, and its sensitivity and specificity calculated. Post hoc analysis was then conducted to assess whether a change in the degree of specificity required for the cut-off value would have materially altered the results.

To measure the effectiveness of serial forms of analysis in the same patient group, the serial PEF data from all records were assessed using the computerised Oasys-2 system. Oasys-2 uses linear discriminant analysis to attempt to reproduce the visual analysis of an expert assessor, and to create a diagnostic score reflecting the likelihood of occupational asthma being demonstrated on a particular record [10]. An Oasys-2 score of $>2.5$ was taken as diagnostic of occupational asthma. Using a second serial technique within the Oasys-2 program, the mean difference between the PEF on work and rest days was also calculated, where a "day" started with the first reading at work and continued to the last reading before work on the following day. A difference in mean PEF between rest and work days of $>16 \mathrm{~L} \cdot \mathrm{min}^{-1}$ was taken as a diagnosis of occupational asthma.

Nonspecific reactivity to methacholine or histamine was measured using the Yan technique [11] during a clinic visit, which took place either the day after $\geqslant 3$ consecutive days at work or on the day after specific challenge. Specific challenge tests were carried out in hospital on an in-patient basis with a suitable control exposure and increasing exposures to the causative agent on separate days. After baseline values, forced expiratory volume in $1 \mathrm{~s}$ (FEV1) was measured at the end of exposure, every $5 \mathrm{~min}$ for the first $30 \mathrm{~min}$, every $10 \mathrm{~min}$ for the next $30 \mathrm{~min}$, and then hourly until bedtime. A positive challenge was taken as a $\geqslant 15 \%$ fall from baseline FEV1 compared with the control exposure.

\section{RESULTS}

Table 1 shows the clinical features of individuals with occupational asthma and asthmatic controls. The occupational asthma and control groups had a mean time between the 


\begin{tabular}{|c|c|c|c|c|}
\hline & \multicolumn{2}{|c|}{ Occupational asthma group } & \multicolumn{2}{|c|}{ Control group } \\
\hline Subjects n & 18 & 18 & 22 & 22 \\
\hline Mean age yrs & 45.2 & 41.4 & 46.9 & 50.2 \\
\hline FEV $1 \%$ pred & $80.5 \pm 26.1$ & $84.4 \pm 23.8$ & $83.7 \pm 20.7$ & $77.6 \pm 15.2$ \\
\hline Nonspecific reactors $\#$ & $12 / 17$ & $11 / 17$ & $6 / 13$ & $7 / 13$ \\
\hline Inhaled corticosteroids & $14 / 17$ & $14 / 17$ & $12 / 13$ & $15 / 17$ \\
\hline Diurnal PEF variation ${ }^{\top} L \cdot \min ^{-1}$ & $26.04 \pm 14.9$ & $18.6 \pm 8.0$ & $23.4 \pm 17.0$ & $22.8 \pm 14.8$ \\
\hline Workdays & $13.6 \pm 8.6$ & $12.4 \pm 5.7$ & $24.6 \pm 11.1$ & $23.0 \pm 7.1$ \\
\hline Oasys-2 score & $2.7 \pm 1.1$ & $3.2 \pm 0.6$ & $1.9 \pm 0.6$ & $1.9 \pm 0.4$ \\
\hline
\end{tabular}

Data are presented as mean $\pm \mathrm{SD}$, unless otherwise stated. Nonspecific reactors and inhaled corticosteroids are given as a proportion of the individuals for whom data was available. FEV1: forced expiratory volume in $1 \mathrm{~s}$; \% pred: \% predicted; PEF: peak expiratory flow. \#: provocative dose of methacholine $(<2,000 \mu \mathrm{g})$ or histamine (<8.0 mg) causing a $20 \%$ fall in FEV 1 ; $"$ : as \% of whole record.

pre- and post-shift readings of $8 \mathrm{~h} 47 \mathrm{~min}$, and $7 \mathrm{~h} 13 \mathrm{~min}$, respectively.

\section{Development set}

The mean \pm SD cross-shift PEF change for those with occupational asthma in the development set was $+1.1 \pm 73$ (range $+190-121.4) \mathrm{L} \cdot \mathrm{min}^{-1}$; for the control group in the development set it was $+19.6 \pm 20.6(+83.2--10.8) \mathrm{L} \cdot \mathrm{min}^{-1}$. The ROC curve (calculated using SPSS Inc., Chicago, IL, USA) for these groups is shown in figure 2 . A cross-shift cut-off value of $-5 \mathrm{~L} \cdot \mathrm{min}^{-1}$ (i.e. a fall from pre- to post-shift PEF of $5 \mathrm{~L} \cdot \mathrm{min}^{-1}$ ) achieved an acceptably high $95.5 \%$ specificity for occupational asthma and a sensitivity of $50 \%$. Figure 3 shows the mean cross-shift changes in PEF for each individual in the study, with the cutoff value at $-5 \mathrm{~L} \cdot \mathrm{min}^{-1}$.

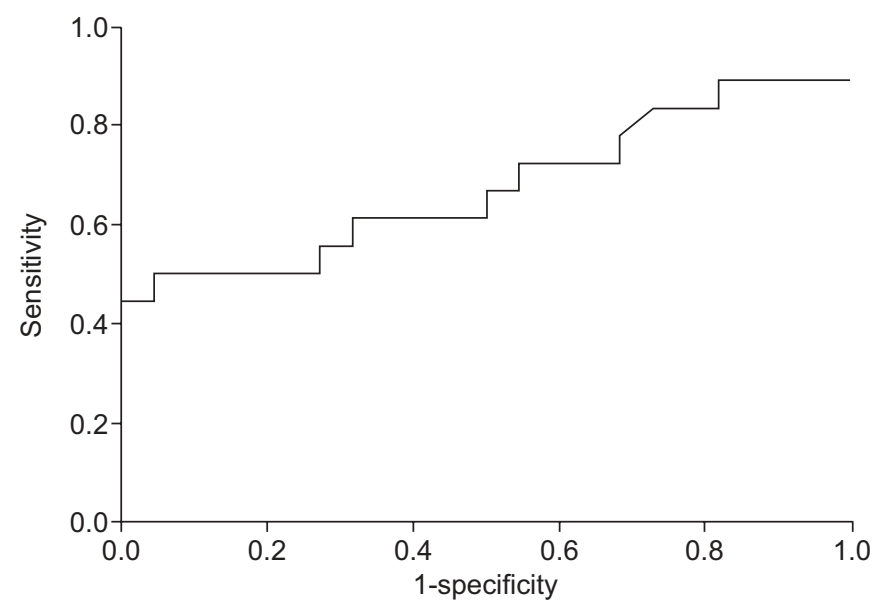

FIGURE 2. The receiver operating curve for cross-shift changes in peak expiratory flow for those with occupational asthma and the controls within the development set (the diagonal segment is produced by a tie in the data).

\section{Evaluation set}

When applied to the evaluation set, the cut-off value of $-5 \mathrm{~L} \cdot \mathrm{min}^{-1}$ correctly identified nine patients from the occupational asthmatic group and inappropriately labelled two patients from the control group as having occupational asthma. This represents a specificity of $90.9 \%$ (95\% CI 0.72 $0.97)$ and sensitivity of $50 \%(95 \%$ CI $0.29-0.71$.) The mean \pm SD cross-shift change in PEF for the individuals in the occupational asthma group was $-0.7 \pm 55.1$ (range $+106.7--100.6$ ) $\mathrm{L} \cdot \mathrm{min}^{-1}$. This indicated that the mean change for the occupational asthma group was a very small fall in PEF between preand post-shift measurements. The equivalent for the control group was $+32.7 \pm 35.7(+108.2--18.9) \mathrm{L} \cdot \mathrm{min}^{-1}$, representing a small average rise in PEF between pre- and post-shift.

Post hoc analysis choosing cut-off values with a range of specificities from the development set and applying them to the evaluation set demonstrated that a reduction in specificity to $\sim 75 \%$ was necessary before the sensitivity improved $>50 \%$.

\section{Serial techniques}

Table 2 shows the performance of cross-shift PEF changes, the Oasys-2 score, and the mean rest-work PEF difference in discriminating the challenge positive occupational asthma group from the control group.

\section{DISCUSSION}

We believe that ours is the only study of cross-shift changes in lung function correlated with specific challenge testing in workers with occupational asthma. The optimum cut-off value derived from our study for simple cross-shift change in PEF had high specificity $(90.9 \%)$ but a poor sensitivity $(50 \%)$. The very small change in PEF represented by our cut-off value (a fall of $5 \mathrm{~L} \cdot \mathrm{min}^{-1}$, below the smallest measurable unit on most PEF devices) was indicative of how little PEF may fall across a morning shift in known occupational asthmatics, emphasising the inappropriateness and impracticalities of cross-shift changes in diagnosis or screening. In the present study, occupational 


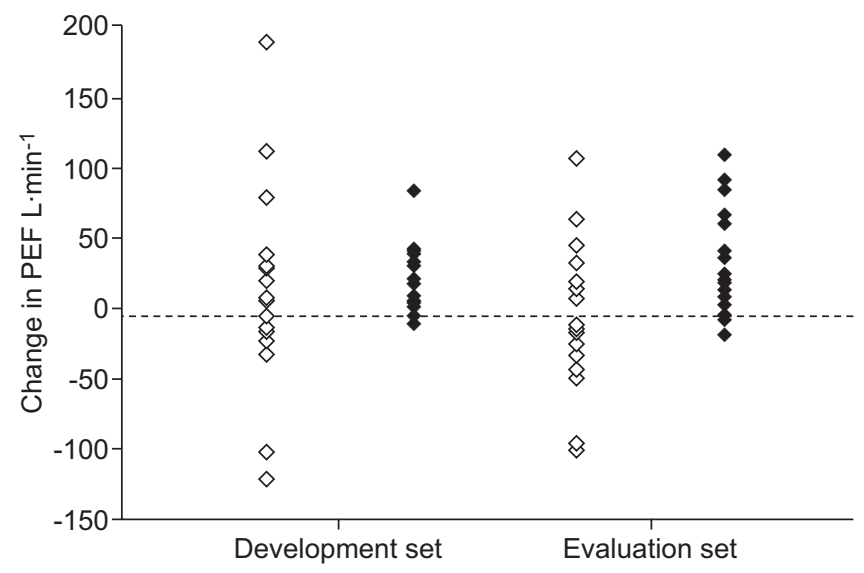

FIGURE 3. The mean cross-shift changes in the peak expiratory flow (PEF) of each individual in the study. The optimum cut-off level (---) was at $-5 \mathrm{~L} \cdot \mathrm{min}^{-1}$. $\diamond$ : working occupational asthmatics; $\bullet$ : asthmatic controls.

asthma could not be excluded by an unchanged PEF or even a rise in its value across a shift. Post hoc analysis demonstrated that a large drop in specificity was required before any significant improvement in sensitivity was measurable. The serial methods that we carried out on the same patient groups provided a much greater degree of accuracy, with the Oasys-2 score achieving a sensitivity of $83.3 \%$ and a specificity of $90.9 \%$, and the work-rest day mean PEF comparison achieving a sensitivity of $66.7 \%$ and a specificity of $100 \%$.

There are many possible explanations as to why a higher sensitivity was not achieved by cross-shift PEF measurement in our study. One likely reason relates to the somewhat simplistic nature of the model that underlies assessment of cross-shift PEF change. For the measurement to be informative, airway calibre in occupational asthma would need to deteriorate soon after exposure to the workplace environment, and then recover quickly overnight allowing a return to baseline for the next morning's pre-shift measurement. This can happen in individuals displaying only an immediate reaction to exposure; however, many other patterns of response are seen. Late reactions occur frequently in occupational asthma and may not become evident for many hours after exposure has commenced; symptoms may not occur until the end of a shift, do not fully recover overnight, and may progressively worsen through the working week $[12,13]$. In a study on occupational asthma in an electronics factory, eight out of 58 workers with wheeze or breathlessness that improved at the weekend described their symptoms as commencing $\geqslant 7 \mathrm{~h}$ after the beginning of their shift. An even larger number were unable to give a precise time for the onset of their symptoms, either because of their irregularity or because they did not recover overnight [14]. Workers with repeated exposure may also reach a stage of relatively fixed airways obstruction and demonstrate little reaction to further stimuli. This is known as a flat reaction. Resolution of symptoms may not occur before several days away from work. Cross-shift measurements taken in this setting will clearly fail to show any significant deterioration.

A further explanation for the relative lack of discrimination has been established in the non-occupational literature. Normal subjects demonstrate a diurnal variation in $\mathrm{PEF}$, with its nadir around waking and a peak $\sim 6 \mathrm{~h}$ later. This diurnal variation is increased in asthmatics [15]. The workers in the present study were all working a morning/day shift, awaking shortly before going to work. Their shift would therefore be expected to coincide with increasing PEF. This would reduce the superimposed effects of any immediate asthmatic reaction following exposure to the work environment, and helps explain why the drop in cross-shift PEF observed in the occupational asthma group was so small. The rise in mean "cross-shift" PEF in the control group subjects represents improvement from their morning dips.

The work-related changes in PEF under investigation in the present study might also be expected to be blunted by medication. Most of the subjects for whom we had data were receiving treatment, including inhaled steroids (table 1.) Data were not available on the use of other therapies, such as inhaled bronchodilators, but it is likely that both groups used these regularly. All were instructed to make serial PEF measurements pre-bronchodilator.

It is worth considering whether measurement of an alternative parameter might have altered our results. FEV1 is frequently measured in cross-shift studies; however, we felt it was unlikely this would be any more discriminating than PEF. Both PEF and FEV1 are good measures of asthma severity and are closely correlated [16]. The inferiority of cross-shift changes in FEV1 in identifying symptomatic subjects as compared to serial changes has been previously reported [17]. PEF has been shown to be as effective as FEV1 in detecting an immediate asthmatic reaction in response to inhaled occupational agents [18]. It is possible that FEV1 is more sensitive in assessing the extent of the late asthmatic reaction, but it may also show more variation from reading to reading [19].

We suggest that in order to counter these confounding factors, and to be sufficiently specific and sensitive for a diagnosis of occupational asthma, PEF measurements need to be carried

TABLE 2 Sensitivity and specificity of different measures of assessment applied to evaluation set data

\begin{tabular}{|c|c|c|c|c|}
\hline & True positives & False positives & Sensitivity \% & Specificity \% \\
\hline Cross-shift fall $>5 \mathrm{~L} \cdot \mathrm{min}^{-1}$ & $9 / 18$ & $2 / 22$ & 50 & 90.9 \\
\hline Mean rest-work PEF $>16 \mathrm{~L} \cdot \mathrm{min}^{-1}$ & $12 / 18$ & $0 / 22$ & 66.7 & 100 \\
\hline
\end{tabular}

True and false positives are given as proportion of individuals in each group. PEF: peak expiratory flow. 
out regularly throughout the day over an extended period of time. Serial analysis can then be carried out by comparison of PEF results at work and at rest. Variation in PEF can be detected across the full working week, rather than just across individual shifts, and account can be taken of underlying diurnal variation and the many different patterns of response shown in workers with occupational exposure. These approaches require some additional input from the subject concerned; however, their increased effectiveness is clearly demonstrated by the present study.

We conclude that whilst the measurement of PEF has an important role in the diagnosis of occupational asthma, isolated cross-shift recordings are greatly limited by a lack of sensitivity.

\section{STATEMENT OF INTEREST}

A statement of interest for P.S. Burge can be found at www.erj. ersjournals.com $/ \mathrm{misc} /$ statements.dtl

\section{REFERENCES}

1 Baur X, Huber H, Degens P, et al. Relation between occupational asthma case history, bronchial methacholine challenge, and specific challenge test in patients with suspected occupational asthma. Am J Ind Med 1998; 33: 114-122.

2 Nicholson P, Cullinan P, Taylor A, et al. Evidence based guidelines for the prevention, identification, and management of occupational asthma. Occup Environ Med 2005; 62: 290-299.

3 Anees W, Gannon P, Huggins V, et al. Effect of peak expiratory flow data quantity on diagnostic sensitivity and specificity in occupational asthma. Eur Respir J 2004; 23: 730-734.

4 Perrin B, Lagier F, L'Archevêque J, et al. Occupational asthma: validity of monitoring of peak expiratory flow rates and nonallergic bronchial responsiveness as compared to specific inhalation challenge. Eur Respir J 1992; 5: 40-48.

5 Côté J, Kennedy S, Chan-Yeung M. Sensitivity and specificity of PC20 and peak expiratory flow rate in cedar asthma. J Allergy Clin Immunol 1990; 85: 592-598.
6 Liss G, Bernstein D, Moller D, et al. Pulmonary and immunologic evaluation of foundry workers exposed to methylene diphenyldiisocyanate (MDI). J Allergy Clin Immunol 1988; 82: 55-61.

7 Enarson D, Vedal S, Chan-Yeung M. Fate of grainhandlers with bronchial hyperreactivity. Clin Invest Med 1988; 11: 193-197.

8 Gee J, Morgan W. A 10-year follow-up study of a group of workers exposed to isocyanates. J Occup Med 1985; 27: 15-18.

9 Orford R, Wilson J. Epidemiologic and immunologic studies in processors of the king crab. Am J Ind Med 1985; 7: 155-169.

10 Gannon P, Newton D, Belcher J, et al. Development of OASYS-2: a system for the analysis of serial measurement of peak expiratory flow in workers with suspected occupational asthma. Thorax 1996; 51: 484-489.

11 Yan K, Salome C, Woolcock A. Rapid method for measurement of bronchial responsiveness. Thorax 1983; 38: 760-765.

12 Burge P, O'Brien I, Harries M. Peak flow rate records in the diagnosis of occupational asthma due to isocyanates. Thorax 1979; 34: 317-323.

13 Burge $\mathrm{P}$, O'Brien I, Harries M. Peak flow rate records in the diagnosis of occupational asthma due to colophony. Thorax 1979; 34: 308-316.

14 Burge P, Perks W, O'Brien I, et al. Occupational asthma in an electronics factory: a case control study to evaluate aetiological factors. Thorax 1979; 34: 300-307.

15 Hetzel M, Clark T. Comparison of normal and asthmatic circadian rhythms in peak expiratory flow rate. Thorax 1980; 35: 732-738.

16 Troyanov S, Ghezzo H, Cartier A, et al. Comparison of circadian variations using FEV1 and peak expiratory flow rates among normal and asthmatic subjects. Thorax 1994; 49: 775-780.

17 Burge P. Single and serial measurements of lung function in the diagnosis of occupational asthma. Eur J Respir Dis Suppl 1982; 123: 47-59.

18 Weytjens K, Malo J, Cartier A, et al. Comparison of peak expiratory flows and FEV1 in assessing immediate asthmatic reactions due to occupational agents. Allergy 1999; 54: 621-625.

19 Leroyer C, Perfetti L, Trudeau C, et al. Comparison of serial monitoring of peak expiratory flow and FEV1 in the diagnosis of occupational asthma. Am J Respir Crit Care Med 1998; 158: 827-832. 